\title{
Morphological component analysis for sparse regularization in plane wave imaging
}

\author{
Adrien Besson*, Rafael E. Carrillo*, Dimitris Perdios*, Eric F. Bezzam ${ }^{\dagger}$, Marcel Arditi*, Yves Wiaux ${ }^{\ddagger}$, \\ and Jean-Philippe Thiran*§ \\ *Signal Processing Laboratory (LTS5), Ecole Polytechnique Fédérale de Lausanne, Lausanne, Switzerland \\ ${ }^{\dagger}$ Audiovisual Communications Laboratory (LCAV), Ecole Polytechnique Fédérale de Lausanne, Lausanne, Switzerland \\ $\ddagger$ Institute of Sensors, Signals and Systems, Heriot-Watt University, Edinburgh, UK \\ ${ }^{\S}$ Department of Radiology, University Hospital Center (CHUV) and University of Lausanne (UNIL), Lausanne, Switzerland
}

\begin{abstract}
Classical ultrasound image reconstruction mainly relies on the well-known delay-and-sum (DAS) beamforming for its simplicity and real-time capability. Sparse regularization methods propose an alternative to DAS which lead to a better inversion of the ill-posed problem resulting from the acoustic wave propagation. In the following work, a new sparse regularization method is proposed which includes a component-based modelling of the radio-frequency images as well as a pointspread-function-adaptive sparsity prior. The proposed method, evaluated on the PICMUS dataset,outperforms the classical DAS in terms of contrast and resolution.

Index Terms-Plane wave, Sparse regularization, Compressed sensing
\end{abstract}

\section{INTRODUCTION}

Classical ultrasound (US) imaging relies on the wellknown Delay-And-Sum (DAS) beamforming which is based on compensating the hyperbolic travel-time delays induced by the propagation from any inhomogeneity in the imaged medium to receiving transducer-elements. DAS is used in most ultrasound modalities due to its ability to be computed in real time. Indeed, DAS beamforming is highly parallelizable and requires only a few delay calculations per pixel. However, it has been demonstrated that it is an approximate solution only to the ill-posed problem of inverting the propagation to retrieve an image [1].

Sparse regularization methods offer an alternative to DAS in which the solution of the inverse problem is retrieved by means of an optimization algorithm. The idea behind these approaches resides in assuming that the image under scrutiny is sparse under a given model. Following this model, the optimization problem searches for the sparsest solution under the data fidelity constraint.

In US imaging, sparse regularization has been extensively used with various models for the sparsity priors. Liebgott et al. [2] use the sparsity of backscattered echoes in the wave atom frame to reduce the number of required echoes in a pre-beamforming step. In a post-beamforming step, a sparsity prior of radio-frequency (RF) images has been exploited in various models such as: Fourier basis [3], wavelet basis [4], sparsity averaging model [5], [6], $\alpha$-stable distribution [7] or more recently in learned dictionaries [8].

In previous works, we have shown that sparse regularization can outperform classical approaches in terms of image qual- ity [5], [9], [10]. However a few key limitations of sparse regularization degrade the quality of the reconstruction in certain cases. Firstly, the use of a single prior for the whole image is intrinsically incompatible with US imaging, where the point-spread-function (PSF) varies spatially across the image. Secondly, modelling the complexity of RF images with only one sparsifying model is an oversimplified approach as it does not take into account the different structures that may appear.

In this paper, we propose a more elaborated sparse regularization approach, based on dividing the desired image into two components, namely a bright reflector part and a background part [11], [12]. The two components are sparse under different models, in a similar manner as described in the morphological component analysis framework (MCA) [13]. In addition, a PSF-adaptive prior is also proposed. Section II reviews the sparse regularization method. The proposed approach is described in Section III and evaluated on the PICMUS dataset in Section IV. Concluding remarks are given in Section V.

\section{SPARSE REGULARIZATION FOR ULTRASOUND IMAGING}

\section{A. General framework}

Sparse regularization methods aim at proposing an alternative to classical image reconstruction methods. Let us denote as $r \in \mathbb{R}^{M}$ the vectorized element raw RF-echo data and as $s \in \mathbb{R}^{N}$ the vectorized RF image. The idea behind sparse regularization is to exploit sparsity of the RF image in a given model in order to invert an ill-posed problem. Thus, it relies on two pillars:

- The ability to express an operator $\mathrm{H} \in \mathbb{R}^{M \times N}$ such that $\boldsymbol{r}=\mathrm{H} \boldsymbol{s}+\boldsymbol{n}$, where $\mathrm{H}$ denotes the measurement operator and $\boldsymbol{n}$ is the noise.

- The ability to find $\Psi \in \mathbb{R}^{N \times J}$ such that $\Psi^{\dagger} s$ is sparse, where $\psi^{\dagger}$ denotes the adjoint of $\psi$.

The desired image is then reconstructed by solving the following analysis-based convex problem:

$$
\min _{\overline{\boldsymbol{s}} \in \mathbb{C}^{N}}\left\|\Psi^{\dagger} \bar{s}\right\|_{1} \text { subject to }\|\boldsymbol{r}-\mathrm{H} \overline{\boldsymbol{s}}\|_{2} \leq \epsilon .
$$

\section{B. Measurement operator in plane wave imaging}

The measurement operator associated to plane wave (PW) imaging has been derived in previous works [6], [9]. Formally, let us denote by $r\left(x_{i}, t\right)$ the element raw data received at 
time $t$ by a transducer positioned at $x_{i}$. Let us also define $s(x, z)$ the RF image corresponding to the point in the medium located at $(x, z)$ If we consider a steered plane wave (SPW) insonification with angle $\theta$, it can be demonstrated that the following equation holds:

$$
r\left(x_{i}, t\right)=\iint_{(x, z) \in \Omega\left(x_{i}, t\right)} s(x, z) d x d z,
$$

where $\Omega\left(x_{i}, t\right)=\left\{(x, z) \mid t=t_{R x}\left(x_{i}, x, z\right)+t_{T x}(x, z)\right\}$, in which $t_{R x}\left(x_{i}, x, z\right)=\sqrt{\left(x-x_{i}\right)^{2}+\left(z-z_{i}\right)^{2}} / c$ and $t_{T x}(x, z)=z \cos \theta / c+x \sin \theta / c$ denote the delays on receive and transmit respectively. The discretization of Equation (2) on the two grids defined by the element raw data and the desired image leads to the following inverse problem:

$$
\boldsymbol{r}=\mathrm{H} \boldsymbol{s}+\boldsymbol{n} .
$$

\section{Sparsity prior}

Various sparsifying models have already been proposed that rely mainly on wavelet-based models [1], [4], [14]. In our previous works [5], [6], [9], [15], we suggested to use a concatenation of wavelet bases since it exhibits better reconstruction results than traditional wavelet-based models. The model, called sparsity averaging (SA) model, is composed of the concatenation of Daubechies wavelet transforms with different wavelet mother functions ranging from Daubechies 1 (Db1) to Daubechies 8 (Db8). Thus,

$$
\Psi=\frac{1}{\sqrt{q}}\left[\Psi_{1}, \ldots, \Psi_{\mathrm{q}}\right]
$$

where $q=8$ and $\Psi_{\mathrm{i}}$ denotes $i^{\text {th }}$ Daubechies wavelet.

\section{Limitations of sparse regularization}

It has been demonstrated that the sparse regularization approach can outperform classical approaches in terms of image quality. However, the methods described above suffer from two main limitations, which are intrinsic to US images. Firstly, ultrasound images are characterized by spatially varying statistics. This aspect is visible in the shape of the PSF which varies in both the lateral and the axial dimensions. Such a variation is not in line with the sparsity prior that assumes the same statistics in the whole image. This leads to problems in the reconstruction of fully-developed speckle [10]. In addition, US images are composed of various components such as fully-developed speckle, bright reflectors and structural parts e.g. carotid arteries, which are each sparse under a different model. Assuming a sparsity prior in a single model is thus inadequate in its ability to reconstruct the variety of complex structures that could be present in a US image.

\section{PROPOSED APPROACH}

In this section, problem (1) is generalized to:

$$
\min _{\overline{\boldsymbol{s}} \in \mathbb{C}^{N}} f(\overline{\boldsymbol{s}}) \text { subject to }\|\boldsymbol{r}-\mathrm{H} \overline{\boldsymbol{s}}\|_{2} \leq \epsilon,
$$

where the prior function $f($.$) will be defined in the following$ subsections.

\section{A. Morphological component analysis}

The first improvement resides in modelling the RF image as a sum of a background and a bright reflector components. Such an approach has raised significant interest in US imaging [11], [12], [16]. This may be formulated as $s=s_{b}+s_{r}$, where $s_{b}$ and $s_{r}$ denote the background and the bright reflector components respectively. Each component is sparse in a given model denoted by $\Psi_{\mathrm{b}}$ and $\Psi_{\mathrm{r}}$ respectively. This leads to the following prior function:

$$
f\left(s_{b}, s_{r}\right)=\left\|\Psi_{\mathrm{b}} s_{b}\right\|_{1}+\left\|\Psi_{\mathrm{r}} s_{\boldsymbol{r}}\right\|_{1} .
$$

Problem (5), with the prior function defined in Equation (6), is similar to the MCA problem [13].

\section{B. Adaptive sparsity prior}

The prior function defined in Equation (6) encapsulates a component-based model of the RF image. However, it does not take into account the spatial variability of the PSF. In order to include such a feature, several assumptions on the behaviour of the PSF are achieved. First, it is assumed that the PSF is spatial-shift invariant within localized parts of the RF image. Secondly, it is supposed that the apodization compensates for the lateral variability of the PSF at a given depth [17]. Taking into account the above assumptions, quasi-stationary regions are obtained by splitting the RF image into non-overlapping segments in the axial dimension [17]. In order to make the algorithm adaptive, a sparsity prior is defined for each region.

Formally, if $s_{b}$ and $s_{\boldsymbol{r}}$ are divided into $L$ non-overlapping segments, the prior function becomes:

$$
f\left(\boldsymbol{s}_{\boldsymbol{b}}, \boldsymbol{s}_{\boldsymbol{r}}\right)=\sum_{i=1}^{L}\left(\left\|\Psi_{\mathrm{b}} \boldsymbol{s}_{\boldsymbol{b}_{\boldsymbol{i}}}\right\|_{1}+\left\|\Psi_{\mathrm{r}} \boldsymbol{s}_{\boldsymbol{r}_{\boldsymbol{i}}}\right\|_{1}\right),
$$

where $\boldsymbol{s}_{\boldsymbol{b}_{\boldsymbol{i}}}$ and $\boldsymbol{s}_{\boldsymbol{r}_{\boldsymbol{i}}}$ correspond to the components of the $i^{\text {th }}$ segment with $i \in\{1, \ldots, L\}$.

Instead of using the $\ell_{1}$-norm as a sparsity promoting norm, the $\ell_{p}$-norm, with $p<1$, is considered in the proposed method. This choice is justified by the fact that it better approximates the $\ell_{0}$-norm. Moreover, a closed-form expression for the shrinkage operator associated with the power $p$ of the $\ell_{p}$-norm has been derived recently by Woodworth et al. [18]. Including this consideration into Equation (7) leads to the following formulation of the prior function:

$$
f\left(\boldsymbol{s}_{\boldsymbol{b}}, \boldsymbol{s}_{\boldsymbol{r}}\right)=\sum_{i=1}^{L}\left(\left\|\Psi_{\mathrm{b}} \boldsymbol{s}_{\boldsymbol{b}_{\boldsymbol{i}}}\right\|_{p}^{p}+\left\|\Psi_{\mathrm{r}} \boldsymbol{s}_{\boldsymbol{r}_{\boldsymbol{i}}}\right\|_{p}^{p}\right) .
$$

\section{The proposed problem}

Given the function defined in Equation (8), it is possible to derive the following problem:

$$
\min _{\overline{\boldsymbol{s}_{\boldsymbol{b}}}, \overline{\boldsymbol{s}_{\boldsymbol{r}}}} f\left(\overline{\boldsymbol{s}_{\boldsymbol{b}}}, \overline{\boldsymbol{s}_{\boldsymbol{r}}}\right) \text { subject to }\left\|\boldsymbol{r}-\mathrm{H}\left(\overline{\boldsymbol{s}_{\boldsymbol{b}}}+\overline{\boldsymbol{s}_{\boldsymbol{r}}}\right)\right\|_{2} \leq \epsilon .
$$

Problem (9) is a classical analysis-based problem, solved using the Douglas-Rachford algorithm [19]. 


\section{Adjustable image display}

The solution of Problem (9) provides the two components $s_{b}$ and $s_{r}$. In order to control the relative amount of background and bright reflector in the desired image, an approach similar to the one proposed by Szasz et al. [12] is used. A parameter $\gamma \in[0,1]$ is introduced such that:

$$
\boldsymbol{s}_{\boldsymbol{d}}=\gamma \boldsymbol{s}_{\boldsymbol{r}}+(1-\gamma) \boldsymbol{s}_{\boldsymbol{b}}
$$

A high value of $\gamma$ favours the reflector component while a low value of $\gamma$ favours the background component.

\section{REsults}

The proposed approach is evaluated on the PICMUS dataset in the following experiments: $1 \mathrm{PW}, 3$ SPWs and 11 SPWs. $\Psi_{\mathrm{b}}$ is the SA model described in Section II-C and $\Psi_{\mathrm{r}}$ is the Dirac basis. For the contrast experiments, a tapered cosine window $(F=1.75)$ is used on receive. No apodization is used for the resolution experiments. The angles for the 11 SPWs experiments are chosen uniformly across the angle range of $16^{\circ}$. For the 3 SPWs experiments, the angles considered are chosen at $-0.43^{\circ}, 0^{\circ}$ and $0.43^{\circ}$. As a reference, the classical DAS is also computed on the dataset for each experiment.

\section{A. Simulated contrast phantom}

The number of segments are set to $L=6$. Since a threshold for the shrinkage operator has to be fixed for each segment and each component, this leads to estimate 12 thresholds. The values of $\epsilon$ are determined by a grid search and set to $0.89\|\boldsymbol{r}\|_{2}, 0.89\|\boldsymbol{r}\|_{2}$ and $0.9\|\boldsymbol{r}\|_{2}$ for the three experiments, respectively. Since the RF image is mostly composed of fullydeveloped speckle, $\gamma$ is set to 0 . The results, displayed in Figure 1(a), show a major increase of the contrast (around $5 \mathrm{~dB}$ ) compared to classical DAS for the three experiments. A visual assessment of the B-mode images in Figures 2(a) and 2(b) confirms the higher quality of the reconstruction for the proposed approach.

\section{B. Experimental contrast phantom}

The number of segments is set to $L=6$. The values of $\epsilon$ are determined by a grid search and set to $0.92\|\boldsymbol{r}\|_{2}, 0.85\|\boldsymbol{r}\|_{2}$ and $0.89\|\boldsymbol{r}\|_{2}$ respectively. Since there is no bright reflector in the RF image, $\gamma$ is set to 0 . The results, displayed in Figure 1 (a), show a major increase of the contrast (around $5 \mathrm{~dB}$ ) compared to classical DAS for the three experiments.

\section{Simulated resolution phantom}

The number of segments is set to $L=8$. The values of $\epsilon$ are determined by a grid search and set to $0.95\|\boldsymbol{r}\|_{2}, 0.95\|\boldsymbol{r}\|_{2}$ and $0.96\|\boldsymbol{r}\|_{2}$ respectively. Since the RF image is composed of point sources only, $\gamma$ is set to 1 . The results, displayed in Figures 1(b) and 1(c), show a major improvement in both lateral and axial resolution compared to DAS for the three experiments. A visual assessment of the B-mode images in Figures 2(c) and 2(d) confirms the better resolution obtained with the proposed approach.

\section{Experimental resolution phantom}

The number of segments is set to $L=12$. The values of $\epsilon$ are determined by a grid search and set to respectively $0.95\|\boldsymbol{r}\|_{2}, 0.9\|\boldsymbol{r}\|_{2}$ and $0.95\|\boldsymbol{r}\|_{2}$. Since the objective is to improve the resolution, $\gamma$ is set to 1 . The results, displayed in Figures 1(b) and 1(c), show a major improvement in both lateral and axial resolution compared to DAS for the three experiments.

\section{E. Limitations and perspective}

The proposed approach outperforms classical DAS in terms of image quality. However, it suffers from several drawbacks. Firstly, the size of the matrix $\mathrm{H}$ may become prohibitive in the case of compound imaging. This is the main reason why the 75 SPWs experiment was not performed. Secondly, the number of parameters to evaluate is larger than with classical sparse regularization as a threshold value for the shrinkage operator has to be evaluated for each segment and for each component. The number of segments is also a parameter that has to be estimated. Empirically, segments of 10 to $20 \mathrm{~mm}$ appear to be a good choice [16]. In addition, the proposed approach is computationally heavy since the reconstruction algorithm is sub-iterative. Indeed, the projection on the $\ell_{2}$-ball of the constraint requires several iterations. For compounding experiments with a high number of SPWs, it makes the problem hardly tractable. A solution to such a problem would be to focus on primal dual algorithms [20].

\section{CONCLUSION}

In this study, we describe a novel sparse regularization method which outperforms classical approaches in terms of contrast and resolution. A component-based model is introduced in which RF images are divided into bright reflector and background components, sparse in two different models. Spatial variability of the PSF is taken into account as the US images are segmented in stripes in which the PSF is assumed to be quasi-stationary. The sparsity prior is then adapted to each stripe, resulting in an unbiased optimization algorithm and a higher image quality.

\section{ACKNOWLEDGEMENTS}

This work was supported in part by the UltrasoundToGo RTD project (no. 20NA21 145911), evaluated by the Swiss NSF and funded by Nano-Tera.ch with Swiss Confederation financing.

\section{REFERENCES}

[1] G. David, J.-1. Robert, B. Zhang, and A. F. Laine, "Time domain compressive beam forming of ultrasound signals," J. Acoust. Soc. Am. vol. 137, no. 5, pp. 2773-2784, 2015.

[2] H. Liebgott, R. Prost, and D. Friboulet, "Pre-beamformed RF signal reconstruction in medical ultrasound using compressive sensing," Ultrasonics, vol. 53, no. 2, pp. 525-533, 2013.

[3] C. Quinsac, A. Basarab, and D. Kouamé, "Frequency domain compressive sampling for ultrasound imaging," Adv. Acoust. Vib., vol. 2012, 2012.

[4] M. F. Schiffner and G. Schmitz, "Fast pulse-echo ultrasound imaging employing compressive sensing," IEEE Int. Ultrason. Symp., 2011. 


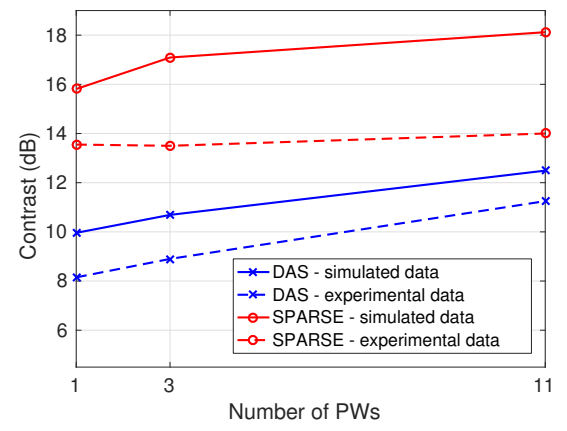

(a)

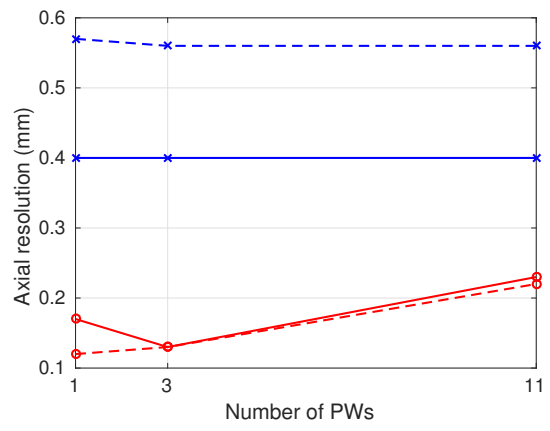

(b)

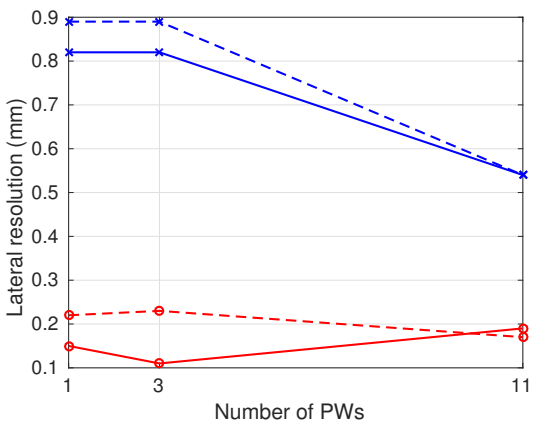

(c)

Figure 1 (a) Contrast, (b) axial resolution and (c) lateral resolution on simulated (solid line) and experimental (dashed line) PICMUS datasets for the classical DAS (blue cross) and the proposed method (red circle) for 1, 3 and 11 PWs.

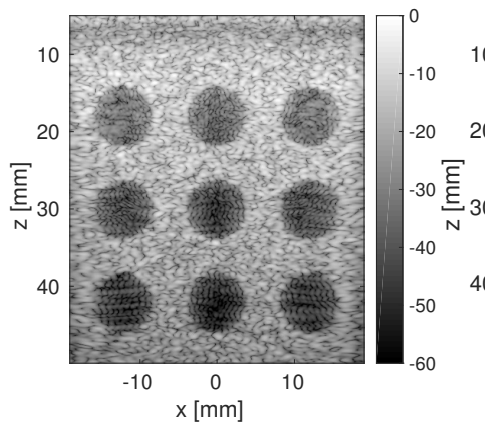

(a)

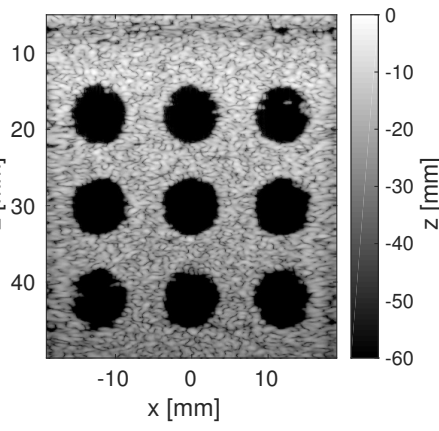

(b)

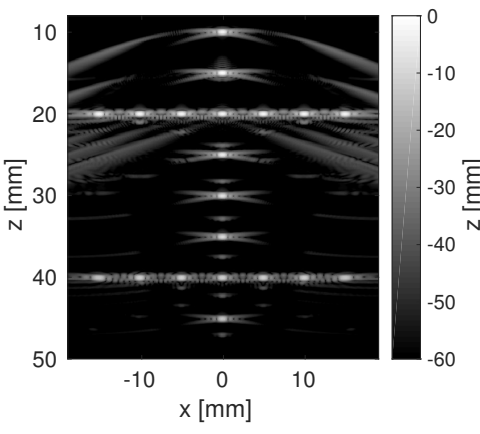

(c)

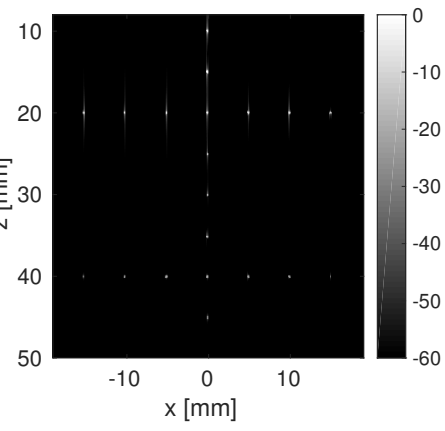

(d)

Figure 2 B-mode images for 1 PW insonification computed on the simulated contrast phantom reconstructed with (a) DAS and (b) the proposed approach, and on the simulated resolution phantom reconstructed with (c) DAS and (d) the proposed approach.

[5] R. E. Carrillo, A. Besson, M. Zhang, D. Friboulet, Y. Wiaux, J.-P. Thiran, and O. Bernard, "A sparse regularization approach for ultrafast ultrasound imaging," in IEEE Int. Ultrason. Symp., 2015.

[6] A. Besson, R. E. Carrillo, O. Bernard, Y. Wiaux, and J.-P. Thiran, "Compressed delay-and-sum beamforming for ultrafast ultrasound imaging," in IEEE Int. Conf. Image Process., pp. 2509-2513, 2016.

[7] N. Dobigeon, A. Basarab, D. Kouamé, and J. Y. Tourneret, "Regularized bayesian compressed sensing in ultrasound imaging," in Eur. Signal Process. Conf., pp. 2600-2604, 2012.

[8] O. Lorintiu, H. Liebgott, M. Alessandrini, O. Bernard, and D. Friboulet, "Compressed sensing reconstruction of 3D ultrasound data using dictionary learning and line-wise subsampling," IEEE Trans. Med. Imaging, vol. 34, no. 12, pp. 2467-2477, 2015.

[9] A. Besson, R. E. Carrillo, M. Zhang, D. Friboulet, O. Bernard, Y. Wiaux, and J.-P. Thiran, "Sparse regularization methods in ultrafast ultrasound imaging," in Eur. Signal Process. Conf., 2016.

[10] A. Besson, M. Zhang, F. Varray, H. Liebgott, D. Friboulet, Y. Wiaux, J.-P. Thiran, R. E. Carrillo, and O. Bernard, "A sparse reconstruction framework for Fourier-based plane wave imaging," Tech. Rep. EPFLARTICLE-218870, Ecole Polytechnique Fédérale de Lausanne, 2016.

[11] Y. Yankelevsky, Z. Friedman, and A. Feuer, "Component based modeling of ultrasound signals." Preprint, arXiv:1603.00273, 2016.

[12] T. Szasz, A. Basarab, and D. Kouamé, "Strong reflector-based beamforming in ultrasound medical imaging," Ultrasonics, vol. 66, pp. 111124, 2016.

[13] J.-L. Starck, M. Elad, and D. Donoho, "Redundant multiscale transforms and their application for morphological component separation," $A d v$. Imaging Electron Phys., vol. 132, pp. 287-348, 2004.

[14] Z. Chen, A. Basarab, and D. Kouamé, "Compressive deconvolution in medical ultrasound imaging," IEEE Trans. Med. Imaging, vol. 35, no. 3, pp. 728-737, 2016.

[15] R. E. Carrillo, J. D. McEwen, D. Van De Ville, J. P. Thiran, and Y. Wiaux, "Sparsity averaging for compressive imaging," IEEE Signal Process. Lett., vol. 20, no. 6, pp. 591-594, 2013.
[16] B. Zhang, J.-L. Robert, and G. David, "Dual-domain compressed beamforming for medical ultrasound imaging," in IEEE Int. Ultrason. Symp., 2015.

[17] O. Michailovich and D. Adam, "A novel approach to the 2-D blind deconvolution problem in medical ultrasound," IEEE Trans. Med. Imaging, vol. 24, no. 1, pp. 86-104, 2005.

[18] J. Woodworth and R. Chartrand, "Compressed sensing recovery via nonconvex shrinkage penalties," Inverse Probl., vol. 32, no. 7, 2016.

[19] P. L. Combettes and J.-C. Pesquet, "A Douglas-Rachford splitting approach to nonsmooth convex variational signal recovery," IEEE J. Sel. Top. Signal Process., vol. 1, no. 4, pp. 564-574, 2007.

[20] N. Komodakis and J.-C. Pesquet, "Playing with duality: An overview of recent primal-dual approaches for solving large-scale optimization problems," IEEE Signal Process. Mag., vol. 32, no. 6, pp. 31-54, 2015. 